\title{
Carrier trapping and luminescence polarization in quantum dashes
}

\author{
A. Musiał, P. Kaczmarkiewicz, G. Sęk, P. Podemski, P. Machnikowski, and J. Misiewicz \\ Institute of Physics, Wroctaw University of Technology, 50-370 Wroctaw, Poland
}

\author{
S. Hein, S. Höfling, and A. Forchel \\ Technische Physik, Universität Würzburg and Wilhelm Conrad Röntgen-Center \\ for Complex Material Systems, Am Hubland, D-97074 Würzburg, Germany
}

\begin{abstract}
We study experimentally and theoretically polarization-dependent luminescence from an ensemble of quantum-dot-like nanostructures with a very large in-plane shape anisotropy (quantum dashes). We show that the measured degree of linear polarization of the emitted light increases with the excitation power and changes with temperature in a non-trivial way, depending on the excitation conditions. Using an approximate model based on the $\boldsymbol{k} \cdot \boldsymbol{p}$ theory, we are able to relate this degree of polarization to the amount of light hole admixture in the exciton states which, in turn, depends on the symmetry of the envelope wave function. Agreement between the measured properties and theory is reached under assumption that the ground exciton state in a quantum dash is trapped in a confinement fluctuation within the structure and thus localized in a much smaller volume of much lower asymmetry than the entire nanostructure.
\end{abstract}

PACS numbers: 78.67.Hc, 73.21.La

\section{INTRODUCTION}

Quantum dashes (QDashes) are epitaxially grown nanostructures strongly elongated in one of the in-plane directions. They can be spontaneously and preferentially formed in a molecular beam epitaxy process of selfassembled growth of, e.g., InAs on InP substrate (Ref. 1 and references therein). The interest in these structures is partly motivated by their applications in telecommunication lasers due to their higher surface density, favorable emission wavelength, wide spectral tunability, broad gain, and high speed modulation $\underline{1}^{-4}$. The existing theoretical predictions on some quantum dash properties include calculation of their electronic states as well as the transition dipole moments and the resulting optical spectra ${ }^{5-7}$. Experimentally, the optical properties of InAs/InP QDashes have been investigated from the point of view of both the ensemble ${ }^{8}$ and the single object properties $\frac{9,10}{10}$. So far, however, the details of the spatial character of the confining potential and polarization properties of these structures have not been analyzed.

A scanning electron microscopy (SEM) image of a QDash sample, shown in Fig. 1, reveals shape irregularities of these structures in the form of sections with locally increased thickness as well as zig-zag bends. While the shape irregularity is a generic feature of QDash structures, the exact morphology varies from sample to sample (depending on the details of the growth procedure) and either thickness fluctuations or bends can be the dominating irregularity feature $\stackrel{111}{ }$. Both the thick sections and the zig-zag corners $\frac{12}{2}$, as well as the possible composition inhomogeneities, can act as additional trapping centers within the confinement volume of the elongated nanostructure. Such an effect of confining in an effective potential on length scales smaller than the size of the entire nanostructure was observed in V-shaped quantum wires 13 . In quantum dashes, the localized charac-

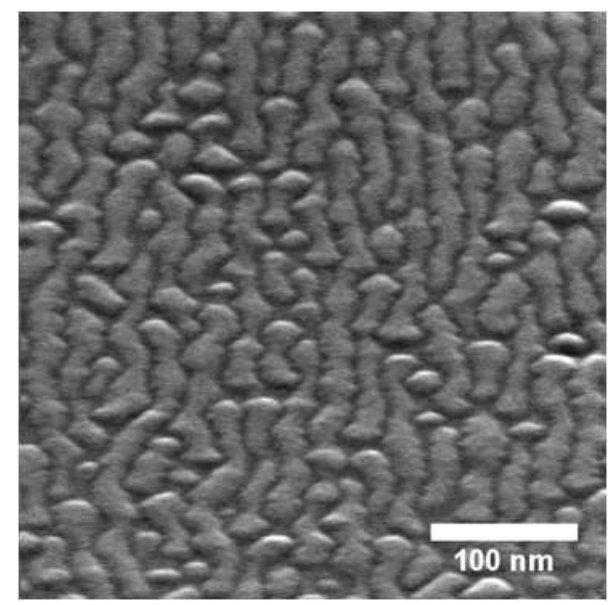

FIG. 1. Scanning electron microscopy image of a QDash sample before overgrowth.

ter of emission at low temperatures in low excitation regime is suggested by experimental observations on single QDashes, where some features typical for the strong confinement regime have been unexpectedly revealed ${ }^{10}$ in spite of the large volume of these structures. First of all, the obtained biexciton binding energy is approximately $0.5 \mathrm{meV}$, which is a value characteristic for very small or very large quantum objects 14 . The latter possibility can, however, be excluded by noting that the estimated exciton to biexciton lifetime ratio is close to 2 , which is a clear fingerprint of a rather strong confinement regime ${ }^{15,16}$.

The presence of an additional confinement within a larger structure can be seen in the temperature dependence of the emission lifetime $\underline{13}$ or in high resolution photoluminescence mapping by using near field optical spectroscopy ${ }^{12}$. However, these methods require time- 
resolved spectroscopy techniques that suffer from the lack of high sensitivity detectors in the $1.55 \mu \mathrm{m}$ range (necessary for the investigation of dashes on the InP substrate) or an AFM-based near field spectroscopy apparatus. Therefore, a different approach for identifying such a two-stage character of the localizing potential in QDash structures is highly desired. Such a method can be based on the fact that the anisotropy of the confinement is reflected in the polarization properties of the luminescence ${ }^{17-22}$. This relation can be traced back to subband mixing effects: Oppositely circularly polarized contributions to the optical emission originating from heavy and light hole transitions interfere, leading to elliptically polarized emission and to the appearance of the preferential orientation of the linear polarization 23,24 . A detailed analysis of the carrier states in an elongated structure, based on the multi-band $\boldsymbol{k} \cdot \boldsymbol{p}$ theory ${ }^{25,26}$ or empirical tight-binding approach 27 indeed reproduces the polarization properties of the observed emission from anisotropic structures. The presumed localizing minima in a QDash can be expected to be significantly more isotropic than the whole QDash itself. Our idea is, therefore, to use the degree of linear polarization of the continuous wave $(\mathrm{cw})$ luminescence of the system as the indicator of the localization of carriers.

In this work, we show that indeed the emission from the states confined in the trapping centers has much more isotropic polarization properties than the radiation originating from states delocalized over the whole QDash volume due to a considerably different degree of anisotropy of their wave functions. For experimental confirmation, we investigate the linear-polarization-resolved photoluminescence (PL) from InAs/InP QDashes for different carrier distributions obtained by changing the temperature and excitation power showing that the polarization study of the QDash luminescence can yield information on the spatial character of the confining potential and reveal the presence of an additional carrier (exciton) trapping within a QDash.

The paper is organized as follows. In Sec. III, we describe the system and the experimental setup and present preliminary PL results motivating the following study. Next, in Sec. IIIA we present a general theory relating the polarization properties to the hole subband mixing, followed by a description of our model of a QDash (Sec. IIIB). Sec. IV presents the results of our polarization-dependent PL measurements and theoretical modeling. The final Sec. $\mathrm{V}$ concludes the paper.

\section{SAMPLE AND EXPERIMENTAL SETUP}

The experiment was performed on an ensemble of selfassembled InAs quantum dashes grown epitaxially on a (001) InP substrate with $3.4 \%$ lattice mismatch in the molecular beam epitaxy technique. The QDash layer is surrounded by additional $\operatorname{In}_{0.53} \mathrm{Ga}_{0.23} \mathrm{Al}_{0.24} \mathrm{As}$ quaternary barriers, lattice matched to InP. The resulting nanostructures are significantly elongated in one of the in-plane directions, preferentially in $[1 \overline{1} 0]^{1}$. In Fig. 1, we present an example of a scanning electron microscopy image of a QDash sample. It should be kept in mind, however, that exact morphology of these structures may vary depending on the growth conditions (see Refs. 1 and 11). The lateral dimensions, estimated for a sample as in Fig. 1, are approximately several to tens of nanometers in width and even hundreds of nanometers in length, confirming the expected significant shape asymmetry (lateral aspect ratio above 4). Their height is typically on the order of a few nanometers. This geometry, formed during the self-assembled growth, is a result of a diffusion coefficient anisotropy due to the surface reconstruction. Further growth details can be found elsewhere $\underline{1,3}$. For the experimental studies, dashes obtained by deposition of approximately $1 \mathrm{~nm}$ of InAs have been chosen, yielding typically the room temperature emission in the range of $1.55 \mu \mathrm{m}$. The surface density of these structures in the sample is rather high and exceeds $10^{11} \mathrm{~cm}^{-2}$.

The linear-polarization-resolved photoluminescence measurements were performed in a standard photoluminescence setup. The structures were non-resonantly excited by a continuous wave semiconductor laser at the wavelength of $660 \mathrm{~nm}$. The excitation powers ranging from $P_{0}=100 \mathrm{nW}$ to $10^{4} P_{0}=1 \mathrm{~mW}$ (determined outside the cryostat) were used. The beam was focused onto a spot of approximately $0.01 \mathrm{~mm}^{2}$ on the sample surface. The emitted light was dispersed in a $0.5 \mathrm{~m}$ focal length monochromator and the signal was detected by a liquid nitrogen cooled InGaAs CCD linear detector. The PL spectra were measured for the two orthogonal polarization directions: along and perpendicular to the quantum dash elongation axis. From these measurements, the degree of linear polarization (DOP), defined as

$$
\Pi=\frac{I_{1}-I_{\mathrm{t}}}{I_{1}+I_{\mathrm{t}}},
$$

was determined, where $I_{\mathrm{l}}$ and $I_{\mathrm{t}}$ are the PL intensities for the linear polarization parallel and perpendicular to the dash elongation axis, respectively. In this work, we choose to study the DOP calculated using the intensities at the maximum of the ensemble luminescence peak. Using intensities integrated over the whole spectral range leads to nearly identical results.

A set of photoluminescence spectra, measured at two temperatures for various excitation powers are presented in Fig. 2. For each temperature and excitation power, two emission spectra for the two orthogonal polarization directions ([110] and [1ํㅣ], i.e., perpendicular and parallel to the quantum dash longer in-plane dimension, respectively) were measured. The first set of the spectra (upper panels in Fig. 21) have been obtained at low temperature for three different excitation powers and normalized, while the second set (lower panels) corresponds to a higher temperature. It can be seen that in all the cases the polarization along quantum dashes dominates. However, at low temperatures, the ratio between the in- 


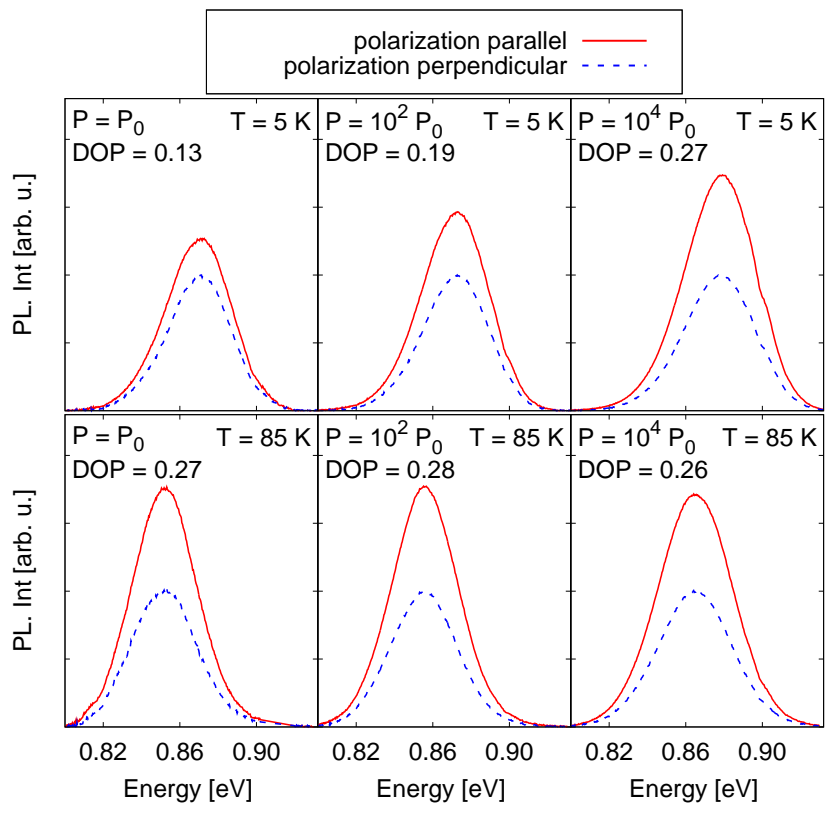

FIG. 2. (Color online) Polarization-resolved photoluminescence spectra measured on the ensemble of InAs/InP quantum dashes for different excitation powers (determined outside the cryostat) and temperatures. The obtained values of the DOP at the peak of the PL spectrum are given in the figures. The spectra are normalized in such a way that the maximum of the signal for the perpendicular polarization is the same for all three powers at a given temperature.

tensities of the linear polarization components increases with the excitation power. As a figure of merit we use the DOP defined in Eq. (11). As can be seen in Fig. 2, the obtained DOP differs by more than a factor of two between the lowest and the highest excitation power used.

Before we proceed to study the polarization-related features in detail let us note that, based on the PL spectra shown in Fig. 2, each QDash appears to behave as an isolated system, that is, coupling between the dashes is negligible. Indeed, if the inter-QDash coupling were strong enough to induce considerable carrier transfer on the time scales of the exciton life time the emission at low excitation powers would be dominated by those dashes in which the exciton energy is the lowest, while higher energy QDashes would contribute at higher excitation due to Pauli blocking (in analogy to the appearance of emission from higher shells in a single nanostructure). This would lead to considerable power-dependent broadening of the ensemble photoluminescence which is not present in the PL spectra (less then $10 \%$ increase of the width is observed between the lowest and highest powers used in Fig. (2).

In addition, only a slight blue shift of the ensemble PL feature is seen in Fig. 2 for increasing powers (about $7 \mathrm{meV}$ between the lowest and the highest powers used). This allows us to exclude heating effects even at the highest powers used, as this would be accompanied with a red shift following the band gap reduction according to the Varshni law. An additional argument against heating effects follows from micro-PL measurements on the same sample $\stackrel{10}{ }$, where much more focused beams, hence much higher power densities are applied. In spite of this, the single QDash lines visible in those experiments show no red shift that would be a signature of heating.

\section{THEORY}

In this section, we first develop a general description of luminescence polarization due to a confinement anisotropy in a nanostructure (Sec. IIIA) and later introduce a specific model of electron, hole and exciton wave functions in a QDash (Sec. IIIB). Although the general theory of Sec. IIIA can be reduced to a simple formula that may be able to qualitatively capture the essential properties of the system one needs a more accurate characterization of the actual wave functions, as proposed in Sec. IIIB in order to quantitatively account for the experimentally observed polarization.

\section{A. Polarization orientation and hole subband mixing}

For the calculation of Coulomb-correlated optically excited states of a semiconductor system it is most common and convenient to use the hole picture, that is, to describe the many-particle states of the almost fully occupied valence band in terms of the few unoccupied states. Single particle (electron and hole) states confined in a nanostructure can then be approximately described in the single-band envelope function approximation by the envelope wave functions $\psi_{\mathrm{c}(\mathrm{v}) i \lambda}(\boldsymbol{r})$, where $\lambda$ denotes the subband, $i$ labels different eigenfunctions of the confinement potential, and $\mathrm{c}(\mathrm{v})$ refers to the conduction (valence) band. Note that in this notation $\psi_{v i \lambda}$ is the hole wave function (rather than an electron wave function for a valence bad state). On the other hand, for multipleband calculations, like the $\boldsymbol{k} \cdot \boldsymbol{p}$-based perturbation theory to be presented below, as well as for a formal discussion of inter-band transitions, it is by far more convenient to use the electron picture, in which the states of electrons in the valence band are directly represented by their wave functions. The hole wave function and the valence band electron wave function are simply related by complex conjugation. Thus, in the electron picture, the envelope wave functions for the valence band electron states are $\psi_{\mathrm{v} i \lambda}^{*}(\boldsymbol{r})$. The corresponding creation and annihilation operators (in the electron picture) will be denoted by $a_{\mathrm{c}(\mathrm{v}) i \lambda}^{\dagger}, a_{\mathrm{c}(\mathrm{v}) i \lambda}$.

If the Coulomb interaction and hole subband mixing is included the energy eigenstates of a confined exciton can 
be written in the general form

$$
\left|X^{(\beta)}\right\rangle=\sum_{\substack{i j \\ \lambda \lambda^{\prime}}} c_{(i \lambda)\left(j \lambda^{\prime}\right)}^{(\beta)} a_{\mathrm{v} i \lambda} a_{\mathrm{c} j \lambda^{\prime}}^{\dagger}|0\rangle
$$

where $\lambda$ and $\lambda^{\prime}$ run through the valence and conduction subbands, respectively, $\beta$ indicates different exciton states, $c_{(i \lambda)\left(j \lambda^{\prime}\right)}^{(\beta)}$ are the coefficients for the expansion in single-subband single-particle states, and $|0\rangle$ is the ground state of the crystal.

The polarization of the light emitted in the recombination process from a conduction band state to a valence band state is determined by the interband matrix element of the positive frequency part of the dipole moment operator $\hat{\boldsymbol{d}}$ (Ref. 28 ),

$$
\hat{\boldsymbol{d}}=\boldsymbol{d}_{\lambda \lambda^{\prime}} \int d^{3} r \psi_{\mathrm{v} i \lambda}(\boldsymbol{r}) \psi_{\mathrm{c} j \lambda^{\prime}}(\boldsymbol{r}) a_{\mathrm{v} i \lambda}^{\dagger} a_{\mathrm{c} j \lambda^{\prime}},
$$

where $\boldsymbol{d}_{\lambda \lambda^{\prime}}$ is the interband matrix element between the states at the $\Gamma$ point of the Brillouin zone in the bulk material (note that, according to our notation, $\psi_{\mathrm{v} i \lambda}(\boldsymbol{r})$ is the complex-conjugated valence band wave function). The dipole moment for the optical transition is therefore

$$
\left\langle 0|\hat{\boldsymbol{d}}| X^{(\beta)}\right\rangle=-\sum_{\lambda \lambda^{\prime}} \boldsymbol{d}_{\lambda \lambda^{\prime}} \alpha_{\lambda \lambda^{\prime}}^{(\beta)}
$$

where

$$
\alpha_{\lambda \lambda^{\prime}}^{(\beta)}=\sum_{i j} c_{(i \lambda)\left(j \lambda^{\prime}\right)}^{(\beta)} \int d^{3} r \psi_{\mathrm{v} i \lambda}(\boldsymbol{r}) \psi_{\mathrm{c} j \lambda^{\prime}}(\boldsymbol{r}) .
$$

Let us assume that the initial state is spin-up $\left(\lambda^{\prime}=\right.$ $+1 / 2)$. The only non-vanishing in-plane components of the dipole moment are to the $+3 / 2$ heavy hole (hh) band and to the $-1 / 2$ light hole (lh) band, with the corresponding bulk matrix elements along the crystallographic $\operatorname{axes}^{28,29}$

$$
\boldsymbol{d}_{3 / 2,1 / 2}=\frac{d_{0}}{\sqrt{2}}\left(\begin{array}{c}
-1 \\
i
\end{array}\right), \quad \boldsymbol{d}_{-1 / 2,1 / 2}=\frac{d_{0}}{\sqrt{6}}\left(\begin{array}{l}
1 \\
i
\end{array}\right) .
$$

Note that the magnitude of the dipole matrix elements, $d_{0}$, is irrelevant for the present study since the DOP is a relative quantity, as follows from Eq. (11).

Since the studied system consists of QDashes elongated in the $[1 \overline{1} 0]$ direction we define unit vectors parallel and transverse to this direction,

$$
\hat{e}_{l}=\frac{\hat{e}_{x}-\hat{e}_{y}}{\sqrt{2}}, \quad \hat{e}_{t}=\frac{\hat{e}_{x}+\hat{e}_{y}}{\sqrt{2}}
$$

where $\hat{e}_{x}, \hat{e}_{y}$ are the unit vectors along the crystallographic axes. From Eqs. (2), (3) and (5), the corresponding components of the interband dipole moment are

$$
\begin{aligned}
d_{l}^{(\beta)} & =\hat{e}_{l} \cdot\left\langle 0|\hat{\boldsymbol{d}}| X^{(\beta)}\right\rangle \\
& =-d_{0} \frac{i+1}{2} \alpha_{3 / 2,1 / 2}^{(\beta)}+d_{0} \frac{1-i}{2 \sqrt{3}} \alpha_{-1 / 2,1 / 2}^{(\beta)}
\end{aligned}
$$

and

$$
\begin{aligned}
d_{t}^{(\beta)} & =\hat{e}_{t} \cdot\left\langle 0|\hat{\boldsymbol{d}}| X^{(\beta)}\right\rangle \\
& =d_{0} \frac{i-1}{2} \alpha_{3 / 2,1 / 2}^{(\beta)}+d_{0} \frac{1+i}{2 \sqrt{3}} \alpha_{-1 / 2,1 / 2}^{(\beta)}
\end{aligned}
$$

If the average occupation of an exciton state with energy $E$ is $n(E)$ then the intensities of light emitted from the state $\beta$, polarized parallel and perpendicular to the direction of the QDash elongation are proportional to $I_{1}^{(\beta)} \sim n\left(E_{\beta}\right)\left|d_{1}^{(\beta)}\right|^{2}$ and $I_{\mathrm{t}}^{(\beta)} \sim n\left(E_{\beta}\right)\left|d_{\mathrm{t}}^{(\beta)}\right|^{2}$, respectively.

In order to provide a description relevant to an inhomogeneously broadened ensemble we consider a set of QDashes with variable parameters that we formally jointly denote by a single symbol $\eta$, representing the full set of relevant parameters. The random distribution of the QDash parameters is represented by the distribution function $f(\eta)$. At this point, the number and the physical nature of the variable parameters as well as the distribution function can be arbitrary but a simple choice will be proposed in Sec. IIIB in order to perform numerical calculations.

For the PL response recorded from an inhomogeneous QDash ensemble at a single emission energy $E$, the PL intensities at the two linear polarizations are then

$$
I_{1 / \mathrm{t}}(E)=\int d \eta f(\eta) \sum_{\beta} n\left(E_{\eta \beta}\right)\left|d_{1 / \mathrm{t}}^{(\eta \beta)}\right|^{2} \delta\left(E_{\eta \beta}-E\right)
$$

where $E_{\eta \beta}$ is the energy of the state $\left|X^{(\beta)}\right\rangle$ and $d_{1 / \mathrm{t}}^{(\eta \beta)}$ are the corresponding components of the dipole moment in a QDash characterized by the parameters $\eta$. According to Eq. (11) and using Eqs. (6) and (7), the ensemble DOP at the emission energy $E$ is then

$$
\Pi(E)=-\frac{2}{\sqrt{3}} \frac{\int d \eta f(\eta) \sum_{\beta} n_{\eta}\left(E_{\eta \beta}\right) \delta\left(E_{\eta \beta}-E\right) \operatorname{Im}\left[\alpha_{3 / 2,1 / 2}^{(\eta \beta) *} \alpha_{-1 / 2,1 / 2}^{(\eta \beta)}\right]}{\int d \eta f(\eta) \sum_{\beta} n_{\eta}\left(E_{\eta \beta}\right) \delta\left(E_{\eta \beta}-E\right)\left[\left|\alpha_{3 / 2,1 / 2}^{(\eta \beta)}\right|^{2}+\frac{1}{3}\left|\alpha_{-1 / 2,1 / 2}^{(\eta \beta)}\right|^{2}\right]}
$$


where $\alpha_{\lambda \lambda^{\prime}}^{(\eta \beta)}$ is the oscillator strength parameter, calculated according to Eq. (4) for a QDash with parameters $\eta$ and $n_{\eta}\left(E_{\eta \beta}\right)$ are the average occupations of the energy levels in such a QDash (occupation redistribution is assumed to be independent for each QDash, that is, no redistribution between the dashes takes place). As can be seen from the above equations, mixing between light and heavy hole states leads to preferential polarization of the emitted light, which depends on the relative phase of the two contributions 23,24 .

In our theoretical modeling, we assume that the lowest hole state consists mostly of a heavy hole component with some admixture from light holes, as is typical for self assembled structures, where the strain is compressive. This is justified in many systems since the light hole states are shifted in energy with respect to the heavy hole states due to confinement and strain, while the inter-subband coupling elements are relatively small. In order to derive the light hole contribution one uses a perturbation theory based on the $\boldsymbol{k} \cdot \boldsymbol{p}$ (Kane) Hamiltonian ${ }^{30}$. As can be seen from the structure of the Kane Hamiltonian, the heavy hole $+3 / 2$ state is coupled in the leading order to both light hole states. However, the exciton state involving a spin $+1 / 2$ electron and a spin $+1 / 2$ hole is dark and does not contribute to the optical properties in the leading order. The calculations of the degree of polarization [Eq. (8)] have been performed with an assumption that coupling to the $+1 / 2$ light hole states can be neglected.

The part of the Kane Hamiltonian relevant to the light hole admixture is

$$
V=\sum_{k l} \int d^{3} r \psi_{\mathrm{v} l, 3 / 2}(\boldsymbol{r}) R \psi_{\mathrm{v} k,-1 / 2}^{*}(\boldsymbol{r}) a_{\mathrm{v} l, 3 / 2}^{\dagger} a_{\mathrm{v} k,-1 / 2},
$$

where $R=R_{\mathrm{k}}+R_{\mathrm{s}}$ is the matrix element of the Kane Hamiltonian coupling the spin $3 / 2$ heavy hole subband with the $-1 / 2$ light hole subband 25,30 . The kinetic contribution to the inter-subband coupling is

$$
R_{\mathrm{k}}=\frac{\sqrt{3} \hbar^{2}}{2 m_{0}}\left[\gamma_{2}\left(k_{x}^{2}-k_{y}^{2}\right)-2 i \gamma_{3} k_{x} k_{y}\right]
$$

where $k_{j}=-i \partial / \partial x_{j}, m_{0}$ is free electron mass and $\gamma_{j}$ are the Luttinger parameters. The strain-induced contribution is

$$
R_{\mathrm{s}}=-\frac{\sqrt{3}}{2} b_{\mathrm{v}}\left(\epsilon_{x x}-\epsilon_{y y}\right)+i d_{\mathrm{v}} \epsilon_{x y},
$$

where $b_{\mathrm{v}}$ and $d_{\mathrm{v}}$ are valence band deformation potentials and $\epsilon_{i j}$ are the strain tensor components. Note that we use the standard definition of the basis functions ${ }^{30}$, consistent with the general theory of the angular momentum ${ }^{31}$, which differs from that used in many papers employing the $\boldsymbol{k} \cdot \boldsymbol{p}$ theory 25,32 .

Assuming the zeroth-order exciton state to be of purely heavy hole type with, say, a spin-up electron,

$$
\left|X^{(\beta, 0)}\right\rangle=\sum_{i j} c_{(i, 3 / 2)(j, 1 / 2)}^{(\beta)} a_{\mathrm{v} i, 3 / 2} a_{\mathrm{cj}, 1 / 2}^{\dagger}|0\rangle,
$$

the first order correction due to the inter-subband coupling is approximated as

$$
\left|X^{(\beta, 1)}\right\rangle=\sum_{k j} c_{(k,-1 / 2)(j, 1 / 2)}^{(\beta)} a_{\mathrm{v} k,-1 / 2} a_{\mathrm{c} j, 1 / 2}^{\dagger}|0\rangle,
$$

with

$$
\begin{aligned}
& c_{(k,-1 / 2)(j, 1 / 2)}^{(\beta)}= \\
& -\frac{1}{\Delta E_{\mathrm{lh}}} \sum_{i} c_{(i, 3 / 2)(j, 1 / 2)}^{(\beta)} \int d^{3} r \psi_{\mathrm{v} i, 3 / 2}(\boldsymbol{r}) R \psi_{\mathrm{v} k,-1 / 2}^{*}(\boldsymbol{r}),
\end{aligned}
$$

where $\Delta E_{\mathrm{lh}}$ is the average energy separation between heavy and light holes. Substituting this to Eq. (4) one finds $\alpha_{-1 / 2,1 / 2}^{(\beta)}=\alpha_{-1 / 2,1 / 2}^{(\beta, \mathrm{k})}+\alpha_{-1 / 2,1 / 2}^{(\beta, \mathrm{s})}$, where $\alpha_{-1 / 2,1 / 2}^{(\beta, \mathrm{k})}$ and $\alpha_{-1 / 2,1 / 2}^{(\beta, \mathrm{s})}$ are the kinetic and strain-induced contributions, respectively, given by

$$
\begin{aligned}
\alpha_{-1 / 2,1 / 2}^{(\beta, x)}= & -\frac{1}{\Delta E_{\mathrm{lh}}} \sum_{i j k} c_{(i, 3 / 2)(j, 1 / 2)}^{(\beta)} \\
& \times \int d^{3} r \psi_{\mathrm{v} i, 3 / 2}(\boldsymbol{r}) R_{x} \psi_{\mathrm{v} k,-1 / 2}^{*}(\boldsymbol{r}) \\
& \times \int d^{3} r \psi_{\mathrm{v} k,-1 / 2}(\boldsymbol{r}) \psi_{\mathrm{c} j, 1 / 2}(\boldsymbol{r}) \\
= & -\frac{1}{\Delta E_{\mathrm{lh}}} \sum_{i j} c_{(i, 3 / 2)(j, 1 / 2)}^{(\beta)} \\
& \times \int d^{3} r \psi_{\mathrm{v} i, 3 / 2}(\boldsymbol{r}) R_{x} \psi_{\mathrm{c} j, 1 / 2}(\boldsymbol{r}),
\end{aligned}
$$

for $x=\mathrm{k}$, $\mathrm{s}$, where we used the completeness relation for the wave functions $\psi_{\mathrm{v} k,-1 / 2}(\boldsymbol{r})$.

As the wave functions can be chosen real, $\alpha_{3 / 2,1 / 2}^{(\beta)}$ is real and only the imaginary part of $\alpha_{-1 / 2,1 / 2}^{(\beta)}$, related to the imaginary (anti-hermitian) parts of $R_{\mathrm{k}}$ and $R_{\mathrm{s}}$, contributes to Eq. (8). In the leading order in the subband-mixing term one then finds the DOP in the form $\Pi=\Pi_{\mathrm{k}}+\Pi_{\mathrm{s}}$, where we split the effect into the kinetic and strain-induced contributions defined by Eq. (8) with $\alpha_{-1 / 2,1 / 2}^{(\eta \beta)}$ replaced in the numerator by its kinetic or strain-related parts $\alpha_{-1 / 2,1 / 2}^{(\eta \beta, x)}, x=\mathrm{k}, \mathrm{s}$, respectively, and neglected in the denominator.

The imaginary (anti-hermitian) part of $R_{\mathrm{k}}$ can be written in the coordinate frame related to the QDash elongation as

$$
\operatorname{Im} R_{\mathrm{k}}=-\frac{\sqrt{3} \hbar^{2}}{2 m_{0}} \gamma_{3}\left(k_{1}^{2}-k_{\mathrm{t}}^{2}\right),
$$

where $k_{1}=\left(k_{x}-k_{y}\right) / \sqrt{2}$ and $k_{\mathrm{t}}=\left(k_{x}+k_{y}\right) / \sqrt{2}$. From Eqs. (8) and (9) it is then clear that the DOP is determined by the matrix elements of $k_{1}^{2}$ and $k_{\mathrm{t}}^{2}$ between the states involved in the optical transition, hence by the symmetry of the wave function.

In order to estimate the strain-related part one would need to know the exact strain distribution in the nanostructure, which is beyond the scope of the present paper. 
Nonetheless, if the localization is due to relatively small and smooth shape fluctuations then the strain field can be expected to vary only very weakly over the volume of the QDash (note that the situation can be completely different if the trapping takes place in corners or bends on the QDash where the strain distribution is likely to be much different from that in the straight segments). Here, we will adopt the assumption of a nearly constant strain field. Then, comparing Eq. (9) for $R_{\mathrm{S}}=$ const with Eq. (4) one immediately gets

$$
\alpha_{-1 / 2,1 / 2}^{(\beta, \mathrm{s})}=-\frac{R_{\mathrm{s}}}{\Delta E_{l h}} \alpha_{3 / 2,1 / 2}^{(\beta)} .
$$

The strain-induced contribution to the DOP in this case is

$$
\Pi_{\mathrm{s}}=\frac{2}{\sqrt{3}} \frac{\operatorname{Im} R_{\mathrm{s}}}{\Delta E_{l h}}
$$

and is the same for every state.

While the result contained in Eqs. (8) and (9) is rather involved and requires a numerical solution for the exciton states, the essential features of our theoretical predictions are already clear in a simplified, semi-quantitative treatment ${ }^{33}$. If the Coulomb coupling is neglected then for a single bright exciton state with both the electron and the hole in the single particle state $i_{0}$ one has $c_{(i, 3 / 2)(j, 1 / 2)}^{(\beta)}=\delta_{i i_{0}} \delta_{j i_{0}}$. In this case, if the electron and hole envelope wave functions are assumed identical then $\alpha_{3 / 2,1 / 2}^{(\beta)}=1$ and

$$
\operatorname{Im} \alpha_{-1 / 2,1 / 2}^{(\beta, \mathrm{k})}=\frac{\sqrt{3} \hbar^{2}}{2 m_{0} \Delta E_{\mathrm{lh}}} \gamma_{3}\left(\left\langle k_{l}^{2}\right\rangle_{i_{0}}-\left\langle k_{t}^{2}\right\rangle_{i_{0}}\right),
$$

where $\langle\ldots\rangle_{i_{0}}$ denotes the average value in the envelope state $i_{0}$. Typically (assuming approximately box-like confinement model),

$$
\left\langle k_{l}^{2}\right\rangle_{i_{0}} \sim \pi^{2} \frac{n_{1}^{2}}{L^{2}} \quad\left\langle k_{t}^{2}\right\rangle_{i_{0}} \sim \pi^{2} \frac{n_{\mathrm{t}}^{2}}{D^{2}}
$$

where $L$ and $D$ are the confinement dimensions along and perpendicular to the QDash, respectively and $n_{\mathrm{l}}, n_{\mathrm{t}}$ are the quantum numbers associated with the excitations along and perpendicular to the QDash elongation. Therefore, from Eq. (8), one can estimate the kinetic contribution to the DOP of light emitted from a single state in this case

$$
\Pi_{\mathrm{k}}\left(n_{\mathrm{l}}, n_{\mathrm{t}}\right)=\frac{\hbar^{2} \pi^{2}}{m_{0} \Delta E_{\mathrm{lh}}} \gamma_{3}\left(\frac{n_{\mathrm{t}}^{2}}{D^{2}}-\frac{n_{1}^{2}}{L^{2}}\right) .
$$

Of course, for other confinement models, the quantum numbers will enter in Eq. (11) in a different way (e.g., linear for a harmonic oscillator). The main conclusions will, nonetheless, be the same.

The approximate formula (11) captures the essential effect of the confinement shape on the polarization properties of the emitted light. For an in-plane isotropic confinement $(L=D)$, there is no preferred polarization axis.
For an anisotropic structure, the light is preferentially polarized along the structure, to the degree dependent on the occupation of the higher excited states. It turns out that this simple model is sufficient to account for the high-temperature spectrally resolved degree of polarization for an ensemble of QDashes 33 . However, for the study of more subtle effects, like additional localization, one needs a more detailed approach, including a realistic confinement shape, the Coulomb correlations, and the thermal distribution of occupations. Therefore, in the following section we introduce a more detailed and accurate model of electron and hole confinement as well as Coulomb-coupled exciton states. While Eq. (11) will be useful for the qualitative interpretation of the results, the general Eq. (8) with the wave functions derived in Sec. IIIB will be used in the numerical modeling of the polarized emission that will be compared with the experimental results.

\section{B. Model of a QDash}

In order to obtain quantitative estimates of the degree of polarization of the radiation emitted by a QDash structure occupied by a single exciton we now introduce a simple model of the system. This will allow us to calculate the kinetic contribution to the polarization, which critically depends on the character of the wave functions. The strain-related part $\Pi_{\mathrm{S}}$ is assumed to be similar for all the states as discussed above. Therefore, this part will be treated as a constant parameter to be found from fitting to experimental results.

Our choice here is to model the carrier trapping by width fluctuations, which are the dominating type of confinement irregularity in some InAs/InP QDash samples 11 . In the Appendix, we show that localization appears also on the zigzag corners. In fact, the exact localization mechanism is not essential for our conclusions. Our choice is convenient for our present purpose as the localization on geometrical fluctuations can be consistently modeled within a simple approach, while trapping on the bends may involve nontrivial strain fields and, therefore, require much more detailed structural modeling, which is beyond the scope of this paper.

The Hamiltonian for a single carrier (electron or hole) confined in a QDash in the single-band effective mass and envelope function approximations is

$$
H_{\alpha}=-\frac{\hbar^{2}}{2 m_{\alpha}^{*}} \Delta+V(\boldsymbol{r})
$$

where $\alpha$ denotes the type of a carrier $(\alpha=\mathrm{e}, \mathrm{h})$. The QDash confinement potential $V(\boldsymbol{r})$ is modeled as a $3 \mathrm{D}$ potential well with the shape reproducing the essential features of the QDash geometry, in particular the presence of a widening that can trap the carriers (see Fig. 3). We assume that the QDash structures have the crosssection in the form of a circular segment, with the base 


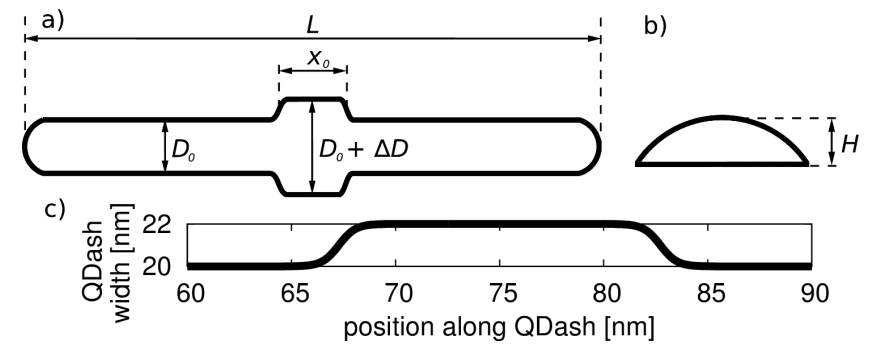

FIG. 3. (a,b) A schematic representation of the QDash geometry used in our model: top (a) and section (b) view. (c) An exact plot of the QDash width as a function of position along its length (only the central section of the structure is shown).

width (chord length) changing along the QDash length (the $x$ coordinate) according to

$$
D(x)=D_{0}+\frac{\Delta D\left(1+4 e^{-b}\right)}{1+4 e^{-b} \cosh \left(2 b x / x_{0}\right)},
$$

where $D_{0}$ is the QDash base width away from the widening, $\Delta D$ is the depth of the fluctuation, $x_{0}$ is the length of the fluctuation, and $b$ defines the shape of the widening (we choose $b=20$; see Fig. [3(c) for the shape of $D(x)$ ). In our model, the widening of the structure is located symmetrically in the center of the QDash (preserving the $D_{2}$ symmetry of the structure). A non-central location of the widening modifies the details of the spectrum due to different selection rules but does not lead to essential modifications of the polarization properties discussed here. The height of the dash is $H(x)=\zeta D(x)$, where $\zeta$ is a constant height to width ratio. For the numerical computations we use standard material parameters for the InAs/InP material pair $\underline{34}$ and parameter values computed for strained nanostructures in this material system ${ }^{35}$ : The effective band offsets between the materials (including strain effects), defining the depth of the confinement potentials, are taken as $400 \mathrm{meV}$ and $250 \mathrm{meV}$ for electrons and holes, respectively. The effective masses used in our calculations are $0.037 m_{0}$ for electrons and $0.33 m_{0}$ for holes, where $m_{0}$ is the free electron mass. The Luttinger parameter is $\gamma_{3}=9.29$.

The approximate single-particle envelope wave functions for electrons and holes are found using a variational method generalizing the "adiabatic" approximation ${ }^{36}$, using the fact that the confinement along the $x$ direction is much weaker than in the other two directions and changes smoothly. First, for each $x$, we variationally minimize the single-particle Hamiltonian (where we suppress the carrier type index $\alpha$ for clarity)

$$
H_{y z}=-\frac{\hbar^{2}}{2 m^{*}}\left(\frac{\partial^{2}}{\partial y^{2}}+\frac{\partial^{2}}{\partial z^{2}}\right)+V(\boldsymbol{r})
$$

in the class of 2-dimensional harmonic oscillator (2DHO)

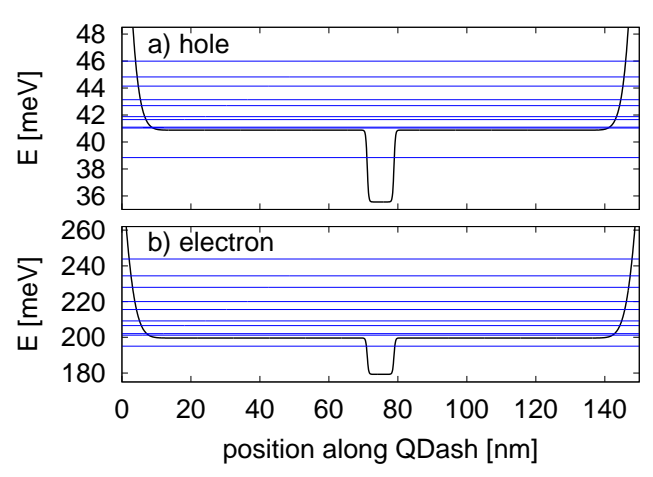

FIG. 4. (Color online) The one-dimensional effective potential along the QDash $\epsilon_{0}(x)$ and the spectrum of single-particle levels for the hole (a) and electron (b).

ground state wave functions

$$
\begin{aligned}
& \psi_{0}(y, z ; x)= \\
& \frac{1}{\sqrt{l_{z}(x) l_{y}(x) \pi}} \exp \left\{-\frac{\left[z-z_{0}(x)\right]^{2}}{2 l_{z}^{2}(x)}-\frac{y^{2}}{2 l_{y}^{2}(x)}\right\},
\end{aligned}
$$

with the variational parameters $l_{y}(x)$ and $l_{z}(x)$ corresponding to the wave function widths in the $y$ and $z$ direction and $z_{0}(x)$ representing the position of the center of the wave function along $z$. Next, we generate the set of $x$-dependent effective potentials

$$
\epsilon_{n}(x)=\int d z \int d y \psi_{n}^{*}(y, z ; x) H_{y z} \psi_{n}(y, z ; x)
$$

where $\psi_{n}(y, z ; x)$ is the wave function of the $2 \mathrm{DHO}$ (with the same parameters as those obtained variationally for the ground state) representing the $n$th state along $y$. This allows us to approximately account for the excited states along $y$ (however, we restrict the dynamics in the strongest confinement direction $z$ to the ground state). The approximate energies $\epsilon_{n}(x)$ are then treated as effective potentials for the one-dimensional eigenvalue equations in the QDash elongation direction,

$$
\left[-\frac{\hbar^{2}}{2 m^{*}} \frac{\partial^{2}}{\partial x^{2}}+\epsilon_{n}(x)\right] f_{n m}(x)=E_{n m} f_{n m}(x)
$$

which are solved numerically (separately for each $n$ ). The full envelope wave functions are then

$$
\psi_{n m}(x, y, z)=\psi_{n}(y, z ; x) f_{n m}(x) .
$$

In the following, both quantum numbers $n$ and $m$ will be denoted by a single index $i$.

In Fig. 4, we show the one-dimensional effective potential along the QDash $\epsilon_{0}(x)$, corresponding to the lowest states in the $y z$ plane, and the spectra of single-particle electron and hole levels. As can be seen, for the parameter values chosen here, there is one trapped state both for the electron and for the hole. 
Based on the calculated single-carrier states, one can construct the product basis for the excitonic states and diagonalize the system described by the Hamiltonian

$$
\begin{aligned}
H= & \sum_{i} E_{i}^{(e)} a_{i}^{\dagger} a_{i}+\sum_{i} E_{i}^{(h)} h_{i}^{\dagger} h_{i} \\
& +\sum_{i j k l} V_{i j k l} a_{i}^{\dagger} h_{j}^{\dagger} h_{k} a_{l},
\end{aligned}
$$

where $a_{i}^{\dagger}, a_{i}$ and $h_{i}^{\dagger}, h_{i}$ are electron and hole creation and annihilation operators, respectively, $E_{i}^{e, h}$ are the energies found from Eq. (14) for electrons and holes, and $V_{i j k l}$ are the matrix elements of the electron-hole interaction,

$$
\begin{aligned}
V_{i j k l}^{e-h}= & \left\langle i j\left|H_{\mathrm{e}-\mathrm{h}}\right| k l\right\rangle \\
= & -\int d^{3} r_{e} \int d^{3} r_{h} \psi_{\mathrm{v} i}^{*}\left(\boldsymbol{r}_{\mathbf{e}}\right) \psi_{\mathrm{c} j}^{*}\left(\boldsymbol{r}_{\mathbf{h}}\right) \\
& \times \frac{e^{2}}{4 \pi \varepsilon \varepsilon_{0}} \frac{1}{\left|\boldsymbol{r}_{\boldsymbol{e}}-\boldsymbol{r}_{\boldsymbol{h}}\right|} \psi_{\mathrm{c} k}\left(\boldsymbol{r}_{\mathbf{h}}\right) \psi_{\mathrm{v} l}\left(\boldsymbol{r}_{\mathbf{e}}\right),
\end{aligned}
$$

where $\varepsilon_{0}$ is the vacuum permittivity and $\varepsilon$ is the relative dielectric constant of the QDash material $(\varepsilon=14.6$ for InAs). Upon the diagonalization of the Hamiltonian given by Eq. (16) one obtains the coefficients $c_{(i, 3 / 2)(j, 1 / 2)}^{(\beta)}$ defining the exciton eigenstates which are then used in the calculation of the DOP according to Eq. (8) (in the leading order in subband mixing).

The inhomogeneous ensemble of QDashes is modeled by assuming a Gaussian distribution of the sizes (corresponding to the formal distribution function $f(\eta)$ introduced in Sec. IIIA) with the standard deviation of $10 \%$ and with a proportional scaling of all the dimensions. The average length of a QDash is taken to be $L=150 \mathrm{~nm}$, the QDash lateral aspect ratio is $L / D_{0}=6$, the height to width ratio is $\zeta=1 / 5.5$, and the size of the width fluctuation is $x_{0}=8 \mathrm{~nm}$, which corresponds to a typical geometry of real structures ${ }^{1,11}$. In our numerical modeling, $\Delta D / D_{0}$ and $\Delta E_{\mathrm{lh}}$ are adjustable parameters.

For the numerical calculations, we choose a set of single carrier states with lowest eigenenergies. In order to achieve convergence we restrict our single carrier states to about 25 electronic states (with the dynamics along the transverse direction restricted to the ground state), and 50 hole states. Since the effective mass of heavy holes is larger than that of an electron, also states with excitations along $y$ direction $(n=2)$, which are coupled to the $n=0$ states by the Coulomb interaction, are included. Depending on the exact values of the QDash shape parameters, the number of states included in the calculations may slightly vary. Luminescence at finite temperatures is calculated using Boltzmann distribution for the occupation of exciton states. This is equivalent to the assumption that thermalization is fast enough to assure full relaxation to equilibrium at the lattice temperature on time scales much shorter than the exciton life time. This should be the case in the system under discussion since the spectrum of a strongly elongated structure is rather dense and the optical response originates from

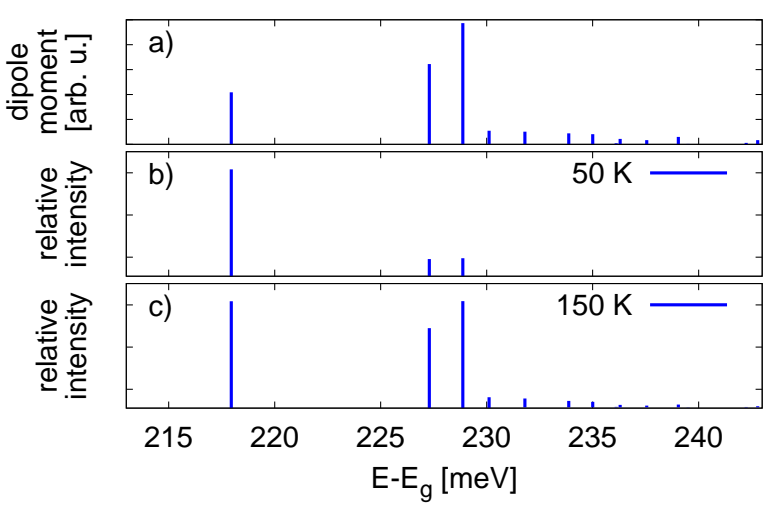

FIG. 5. (Color online) (a) Dipole moments for optical transitions for the exciton states obtained from numerical calculations for a $150 \mathrm{~nm}$ long QDash with $D / D_{0}=0.1$. (b) and (c) Emission spectrum from a single QDash taking into account thermal distribution of the carriers at two different temperatures. Here, relative intensities (normalized to the intensity of the ground state transition) are shown.

structures where electron-hole pairs are captured, which enables the efficient electron-hole scattering-assisted relaxation channels.

In Fig. 5, we show the optical properties of a single QDash obtained from our numerical calculations. Fig. 5(a) presents the interband dipole moments (oscillator strengths) for various exciton states confined in the QDash. In Fig. 5(b,c), the computed emission spectrum at finite temperatures is shown. The lowest energy line corresponds to a state trapped in the width fluctuation while the higher energy lines are related to states delocalized in the whole QDash volume. The lowest delocalized states have larger oscillator strengths than the trapped ground state but because the latter is down-shifted by the trapping and Coulomb energy the contribution from the delocalized states becomes large only at relatively high temperatures. Note, however, that the appearance of the higher energy emission lines in a single QDash spectrum some $10 \mathrm{meV}$ above the fundamental transition does not considerably affect the ensemble emission shown in Fig. 2 because of the large inhomogeneous broadening of about $50 \mathrm{meV}$. As a result, the ensemble emission feature is rather symmetric at all temperatures, reflecting the symmetric ensemble distribution of QDash morphology features, which is common for this material system ${ }^{3}$.

\section{RESULTS AND DISCUSSION}

In this section we present the results of measurements and theoretical modeling that aim at a complete characterization and explanation of the polarization properties of the luminescence from QDashes, as a function of temperature and excitation power. It should be noted that our theory presented in Sec. III is formally limited to 


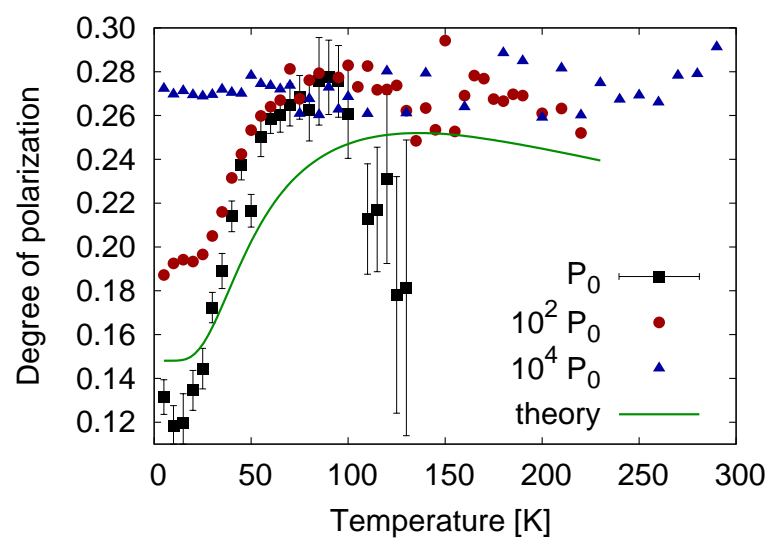

FIG. 6. (Color online) Points: Temperature dependence of emission degree of polarization from the ensemble of InAs/InP quantum dashes in three different excitation regimes as indicated. For clarity, the error bars are shown only for the lowest power data where the measurement uncertainty is the largest. Solid line: the theoretical result.

the low power regime, where a single exciton model for a QDash emission is valid. Taking into account a reasonable estimate of losses, the power density and laser spot size as given in Sec. II corresponds to the photon fluence rate on the order of $10^{6} /\left(\mathrm{cm}^{2} \cdot \mathrm{ns}\right)$. With the average exciton lifetime of $1 \mathrm{~ns}$, one gets an estimate of the QDash occupation on the order of $10^{-5}$ (assuming the exciton lifetime of $1 \mathrm{~ns}$ ) for the lowest excitation power used. Double occupation under weak excitation conditions is therefore highly improbable, even if one allows for the extension of charge life time due to dark spin configurations and trapping of unpaired carriers. Hence, the single-exciton theory provides a basis for the interpretation of the temperature dependence of the DOP at low powers. However, qualitative or semi-quantitative interpretation of power-dependent spectra is also possible, based on the concept of state filling (due to Pauli blocking) and on the general tendencies predicted by Eq. (11).

In order to systematically study the dependence of the DOP on the excitation conditions and temperature we have measured the DOP as a function of temperature for three different excitation powers, shown in Fig. 6. When a very low excitation power is used the DOP at low temperatures is on the level of $0.12-0.13$ and then increases with temperature up to about 0.27 at above $60 \mathrm{~K}$ (which roughly corresponds to $5 \mathrm{meV}$ activation energy). The dependence is similar for the intermediate excitation power but the initial (low temperature) value is higher (about 0.19). Eventually, the DOP is almost constant on the level of 0.27 over the entire temperature range for a very high excitation.

These results are consistent with the emission properties from either locally trapped or delocalized (in the whole QDash volume) states. Indeed, as discussed in Sec. III the DOP for the emission from a given state depends on the imaginary part of the relevant matrix element of the $\boldsymbol{k} \cdot \boldsymbol{p}$ Hamiltonian given by Eq. (10) which, in turn, is determined by the values of the wave vector components $k_{1}, k_{\mathrm{t}}$ (along and perpendicular to the QDash elongation) characteristic for the relevant wave function. At low temperature and low excitation, the detected emission originates mainly from the ground state excitons localized at the QDash potential fluctuations, which are more isotropic than the dash itself. Due to the reduced anisotropy, $k_{1} \sim k_{\mathrm{t}}$, hence the kinetic component to the DOP is small. This results in a small DOP, similar to that observed for slightly asymmetric quantum $\operatorname{dot} 33$. By increasing the temperature, the excitons are thermally released into the whole QDash volume. For such low-energy extended states the confinement along the QDash is much weaker than across the structure. Since $k_{1} \sim 1 / L$ and $k_{\mathrm{t}} \sim 1 / D_{0}$ these two wave vector components become imbalanced and hence the emission becomes more linearly polarized. This effect is also clear from the simplified Eq. (11) if one substitutes for $L$ the effective confinement size for a given state $(L \sim D$ for a trapped ground state, $L \gg D$ for a delocalized higher energy state).

With increasing excitation power, the higher states with anisotropic wave functions become filled and contribute to luminescence already at low temperatures. Therefore, the low temperature DOP increases and the amplitude of the temperature-dependent change is reduced until, for the highest excitation, the contribution of the localized excitons to the emission becomes negligible and the DOP does not significantly change with temperature. Remarkably, the saturation level for the DOP is the same for all the excitation powers, i.e., it is insensitive to the excitation conditions, reflecting the intrinsic asymmetry of the carrier states trapped in the QDash.

The results obtained from the full theoretical model presented in Sec. [II] using the exciton wave functions found in Sec. IIIB and the general equations (9) and (8) for a certain set of parameters are shown by the green solid line in Fig. 6. As our theory is restricted to single-exciton states the theoretical curve corresponds to the low excitation limit. The S-shaped temperature dependence is reproduced if one assumes a QDash ensemble with an appropriately chosen relative amplitude of the widening. For the curve in Fig. 6, $\Delta D / D_{0}=0.1$. For this value of the shape parameter, only the exciton ground state exhibits highly localized properties, which are reflected by a moderate value of the calculated DOP for low temperatures. Clearly, the model is able to reproduce all the qualitative features of the measured dependence: When only the ground state is occupied the degree of polarization of emitted radiation is small. At higher temperatures, emission from higher energy excitonic states (extended over the whole area of a QDash) contributes, leading to the increased value of DOP. The theoretical result shown in Fig. 6, is obtained for the separation between light end heavy holes $\Delta E_{\mathrm{lh}}=20 \mathrm{meV}$ 


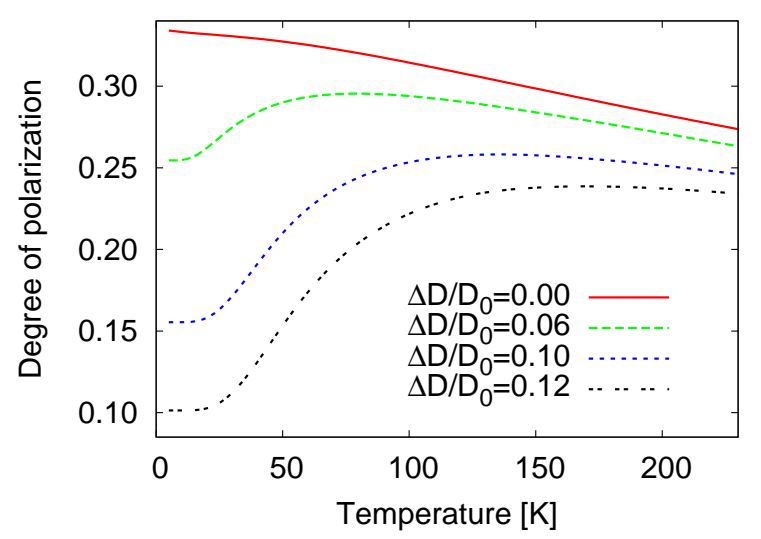

FIG. 7. (Color online) Influence of the amplitude of the widening of a QDash on the calculated DOP of the system. The strain contribution is assumed the same in all the cases.

(the amplitude of the DOP change scales inversely proportional to this parameter). While this value is rather small, one should notice that this energy is an effective parameter averaged over all the heavy hole states contributing to the luminescence and should not be treated as the separation between the lowest heavy hole state and the light hole subband (in the decoupled band picture).

As can be seen, our model is also able to account to some extent for the observed decrease of the DOP at high temperatures for lower excitation powers. This effect can again be understood with the help of the simplified Eq. (11). As the temperature grows, the first process is the release of carriers from the additional confinement center which leads to an increase of the effective confinement length $L$ in Eq. (11) as discussed above. At first, the lowest lying states are filled so that $n_{1} \sim 1$. However, when the temperature further increases higher excited states are occupied with growing values of $n_{\mathrm{l}}$, while $n_{\mathrm{t}}$ remains small due to the stronger confinement and higher excitation energy in the transverse lateral direction. It is clear from Eq. (11) that this increase of $n_{1}$ reduces the value of the DOP as indeed observed in the low-power experimental data and in the theoretical result. Quantitatively, the strong decrease of the low-excitation DOP at high temperatures is not reproduced by the theory probably due to the simplified nature of our QDash model which does not properly account for the states in the higher energy sector. Moreover, one needs to keep in mind that the signal values at these conditions are very low, hence the uncertainty of the measured DOP is very high in this range (see error bars in Fig. 6).

Let us emphasize that the additional localization is essential for the explanation of the observed polarization properties of the PL from QDash structures. The experimental results cannot be reproduced even qualitatively in the absence of additional confinement inside a QDash. When the shape of a QDash is more uniform the ground state of the system is not trapped and has a rather

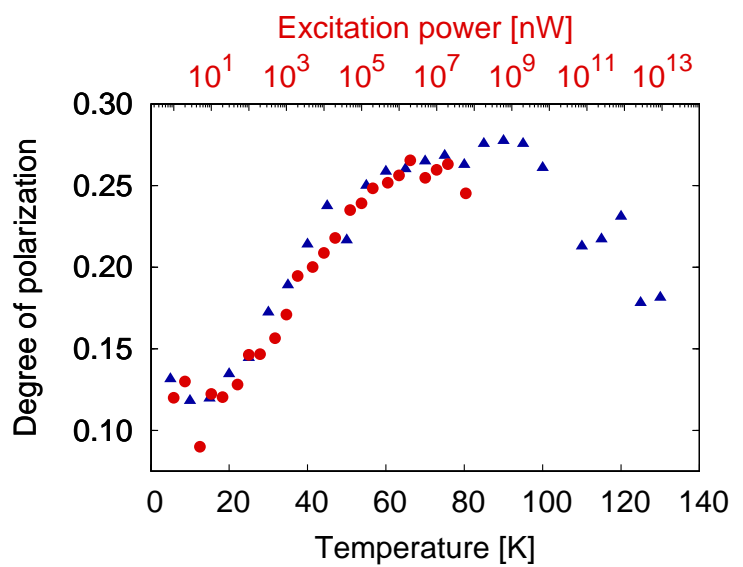

FIG. 8. (Color online) The comparison between the temperature (bottom horizontal axis, blue triangles) and excitation power (top horizontal axis, red circles) dependence of emission degree of polarization for the ensemble of InAs/InP quantum dashes.

anisotropic wave function, similar to all the other states in the structure. As shown in Fig. 7, this results in a relatively high degree of polarization already at low temperatures, which decreases at higher temperatures due to increasing occupation of higher excited states. This kind of behavior is a fingerprint of a nanostructure with a uniform width. When the widening of a QDash is large enough to confine carriers the results change considerably. Thus, the character of polarization properties of emitted radiation reflects the shape of confining potential: Different confinement conditions lead to qualitatively different polarization properties of luminescence from the system. Interestingly, the saturation level and the high-temperature behavior are different for different sizes of the shape fluctuation, which shows that the presence of the trapping potential is important even when the emission from the delocalized states becomes dominant.

Effects similar to those observed in the temperature dependence can be seen in the excitation power dependence of the linear polarization (red circles in Fig. 8). The DOP increases and finally saturates on the same level as in the temperature dependence. Obviously, the cases of high temperature and high excitation power are physically different: In the former, we deal with a single exciton system in which the occupation of higher states is due to thermal redistribution. In the latter, one has a many-body system with excited state occupation forced by state filling. The correspondence between the DOP observed in these two essentially different cases provides a strong confirmation that the effect is due to increased occupation of excited states, which is a common factor of these two physical situations: Since the higher states have anisotropic wave functions they contribute strongly to polarized emission. A straightforward comparison of temperature and excitation power influence on the DOP is presented in Fig. 8 where the DOP axis is common 
and the temperature and excitation power axes (horizontal ones) are translated with respect to each other and scaled in order to emphasize the correspondence between the shapes of the curves. This figure shows directly that the two external factors give a similar effect if the excitation power values are presented in a logarithmic scale. This is consistent with the fact that the occupations of excited states scale with the excitation power according to a power law, while their dependence on temperature is exponential. Therefore, the agreement between the two dependencies supports the idea that the distribution of carriers over the confined states in the QDash is crucial for the polarization properties of the emitted light: Higher excitation power creates more electron-hole pairs which occupy higher energy states according to Pauli exclusion principle, whereas elevated temperatures result in the redistribution of carriers already existing in the system allowing them to occupy higher energy levels. Essentially, however, both mechanisms lead to the observation of radiative recombination of carriers from higherenergy states that are expected to be confined in the whole QDash volume. At the same time, the logarithmic scaling between the temperature and power dependence is a strong argument in favor of state filling as the mechanism of the power dependence, as opposed to heating. If the excitation power acted via heating the local temperature would be proportional to the power and the dependencies on the power and temperature would scale linearly, which is not the case.

\section{CONCLUSIONS}

We have investigated polarization-resolved luminescence from an ensemble of InAs/InP quantum dashes. We have developed a theory relating the observed degree of linear polarization to the asymmetry of the carrier wave functions via the anisotropy effect on the hole subband mixing. By comparing the temperature and power dependent degree of polarization to the theoretical predictions we were able to conclude that the lowest carrier states usually have a much lower degree of asymmetry than the structure itself, which can be related to additional trapping of the excitons to potential fluctuations within the dash volume. Thus, our findings reveal a nontrivial character of carrier states in these systems of technological and applicational relevance.

Our analysis shows, in addition, that the $\mathrm{cw}$ polarization-resolved spectroscopy can be used as a probe of the localization effects within such elongated objects. Thus, this widely available optical tool can yield important information on the actual properties of carrier states in anisotropic systems.

Apart from the general interest in the electronic structure of quantum dashes, the knowledge on their polarization properties and the nature of the carrier states is essential for modeling the emission properties of such nanostructures and can be important for the operation of some optoelectronic devices, especially in those applications where polarization control is important (e.g., polarization-insensitive optical amplifiers ${ }^{38}$ ). The strongly confined character of the lowest carrier states in QDashes will also have a considerable impact on the performance of futuristic photonic devices which are based on quantum electrodynamics experiments ${ }^{39}-42$. It will affect, for instance, the exciton fine structure and anisotropy splitting energies, the energies and even relative spectral position of the excitonic complexes in a QDash, and finally the kinetics of the transitions between the excitonic states. The effect of carrier (exciton) trapping will affect both the total exciton oscillator strength and the polarization selectivity which become crucial when QDashes are used as quasi-zero-dimensional emitters placed inside a microcavity, suitable for single photon sources in the telecommunication wavelength range of $1.3-1.55 \mu \mathrm{m}$.

\section{ACKNOWLEDGMENTS}

This work has been supported by the Polish Ministry of Science and Higher Education within Grant No. N N202 181238 , by the COPERNICUS award of the Foundation for Polish Science and Deutsche Forschungsgemeinschaft, and by the State of Bavaria. PK and PM acknowledge support from the TEAM programme of the Foundation for Polish Science, co-financed by the European Regional Development Fund.

\section{Appendix A: Localization on zig-zag corners}

In this Appendix, we verify that zig-zag bends act as localization centers in a similar way to local widenings. Thus, the theory presented in this paper is applicable also to QDash systems where bends, rather than widenings, are the dominating kind of shape irregularities. Here, we aim at demonstrating the localization effect qualitatively, therefore we present a simple model, including only the geometrical features of the confinement. It should be kept in mind, however, that in a real system a specific strain distribution will occur in the bend area, which must be taken into account in more realistic modeling.

We consider a QDash of dimensions as in the main body of the paper with a homogeneous cross section but with a $90^{\circ}$ bend present in the middle (see the outlines in the upper part of Fig. 91). The electron confinement is modeled as a three-dimensional potential box with the confinement potential depth and cross-section geometry as described in Sec. IIIB. The electron wave functions are found in a way similar to that presented in Sec. IIIB (see also Fig. 3) but this time only the one-dimensional Schrödinger equation along the strongest confinement direction $z$ is solved, yielding a two-dimensional effective potential for the eigenvalue problem in the $x y$ plane. The latter is solved by expansion into plane waves. 

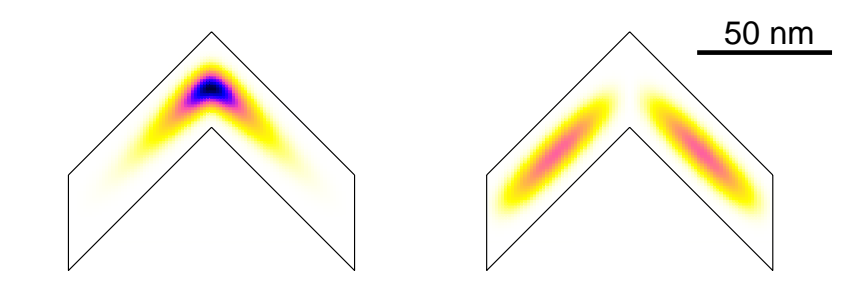

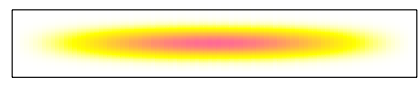

ground state

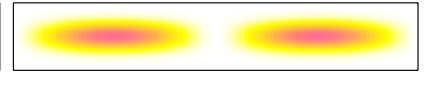

1st excited state
FIG. 9. (Color online) The electron densities in the $x y$ plane (integrated over $z$ ) for the ground and first excited state of a bent and straight dash.
In Fig. 9(a), we show the resulting probability densities for the ground and first excited electron state for the bent dash, as compared with a straight, rectangular dash. It is clear that the ground state gets localized on the corner, while the first excited state remains delocalized in the same way for both the bent and straight QDash.
1 J. P. Reithmaier, G. Eisenstein, and A. Forchel, Proc. IEEE 95, 1779 (2007).

${ }^{2}$ H. S. Djie, Y. Wang, Y.-H. Ding, D.-N. Wang, J. C. M. Hwang, X.-M. Fang, Y. Wu, J. M. Fastenau, A. W. K. Liu, G. T. Dang, W. H. Chang, and B. S. Ooi, Ieee J. Sel. Top. Quant. El. 14, 1239 (2008).

3 A. Sauerwald, T. Kummell, G. Bacher, A. Somers, R. Schwertberger, J. P. Reithmaier, and A. Forchel, Appl. Phys. Lett. 86, 253112 (2005).

4 S. Hein, S. Höfling, and A. Forchel, IEEE Phot. Tech. Lett 21, 528 (2009).

5 P. Miska, J. Even, C. Platz, B. Salem, T. Benyattou, C. Bru-Chevalier, G. Guillot, G. Bremond, K. Moumanis, F. H. Julien, O. Marty, C. Monat, and M. Gendry, J. Appl. Phys. 95, 1074 (2004).

6 J. H. Wei and K. S. Chana, J. Appl. Phys. 97, 123524 (2005).

7 J. Planelles, M. Royo, A. Ballester, and M. Pi, Phys. Rev. B 80, 045324 (2009).

8 W. Rudno-Rudziński, R. Kudrawiec, P. Podemski, G. Sęk, J. Misiewicz, A. Somers, R. Schwertberger, J. P. Reithmaier, and A. Forchel, Appl. Phys. Lett. 89, 031908 (2006).

9 T. Mensing, L. Worschech, R. Schwertberger, J. P. Reithmaier, and A. Forchel, Appl. Phys. Lett. 82, 2799 (2003).

10 G. Sęk, P. Podemski, A. Musiał, J. Misiewicz, S. Hein, S. Höfling, and A. Forchel, J. Appl. Phys. 105, 086104 (2009).

11 B. Ooi, H. Susanto Djie, Y. Wang, C.-L. Tan, J. Hwang, X.-M. Fang, J. Fastenau, A. Liu, G. Dang, and W. Chang, IEEE J. Sel. Top. Quant. El. 14, 1230 (2008).

12 F. Intonti, V. Emiliani, C. Lienau, T. Elsaesser, R. Notzel, and K. H. Ploog, Phys. Rev. B 63, 075313 (2001).

13 T. Guillet, R. Grousson, V. Voliotis, X. L. Wang, and M. Ogura, Phys. Rev. B 68, 045319 (2003).

14 G. A. Narvaez, G. Bester, and A. Zunger, Phys. Rev. B 72, 245318 (2005).

15 G. A. Narvaez, G. Bester, A. Franceschetti, and A. Zunger, Phys. Rev. B 74, 205422 (2006).

16 M. Wimmer, S. V. Nair, and J. Shumway, Phys. Rev. B 73, 165305 (2006).

17 M. Jo, T. Mano, and K. Sakoda, Appl. Phys. Express 3, 045502 (2010).

18 T. Mensing, S. Reitzenstein, A. Loeffler, J. P. Reithmaier, and A. Forchel, Physica E 32, 131 (2006).

19 P. Ridha, L. Li, A. Fiore, G. Patriarche, M. Mexis, and P. M. Smowton, Appl. Phys. Lett. 91, 191123 (2007).

20 P. Podemski, G. Sęk, K. Ryczko, J. Misiewicz, S. Hein, S. Höfling, A. Forchel, and G. Patriarche, Appl. Phys. Lett. 93, 171910 (2008).

21 P. Yu, W. Langbein, K. Leosson, J. M. Hvam, N. Ledentsov, D. Bimberg, V. M. Ustinov, A. Y. Egorov, A. E. Zhukov, A. F. Tsatsul'nikov, and Y. G. Musikhin, Phys. Rev. B 60, 16680 (1999).

22 C. Tonin, R. Hostein, V. Voliotis, R. Grousson, A. Lemaitre, and A. Martinez, arXiv:1110.4042v1 (unpublished).

23 A. V. Koudinov, I. A. Akimov, Y. G. Kusrayev, and F. Henneberger, Phys. Rev. B 70, 241305 (2004).

24 Y. Léger, L. Besombes, L. Maingault, and H. Mariette, Phys. Rev. B 76, 045331 (2007).

25 J. Andrzejewski, G. Sęk, E. O'Reilly, A. Fiore, and J. Misiewicz, J. Appl. Phys. 107, 073509 (2010).

26 T. Saito, H. Ebe, Y. Arakawa, T. Kakitsuka, and M. Sugawara, Phys. Rev. B 77, 195318 (2008).

27 W. Sheng and S. J. Xu, Phys. Rev. B 77, 113305 (2008).

28 H. Haug and S. W. Koch, Quantum Theory of the Optical and Electronic Properties of Semiconductors, 4th ed. (World Scientific, Singapore, 2004).

29 V. M. Axt, Ph.D. thesis, Rheinisch-Westfälische Technische Hochschule Aachen, 1994.

30 R. Winkler, Spin-Orbit Coupling Effects in TwoDimensional Electron and Hole Systems, Vol. 191 of Springer Tracts in Modern Physics (Springer, Berlin, 2003).

31 J. J. Sakurai, Modern Quantum Mechanics (Addison Wesley, Reading, Massachusetts, 1994).

32 C. Pryor, Phys. Rev. B 57, 7190 (1998).

33 P. Kaczmarkiewicz, A. Musiał, G. Sęk, P. Podemski, P. Machnikowski, and J. Misiewicz, Acta Phys. Pol. A 119, 633 (2011).

34 P. Lawaetz, Phys. Rev. B 4, 3460 (1971).

35 M. Holm, M.-E. Pistol, and C. Pryor, J. Appl. Phys. 92, $932(2002)$.

36 A. Wójs, P. Hawrylak, S. Fafard, and L. Jacak, Phys. Rev. B 54, 5604 (1996). 
37 S. Cortez, O. Krebs, P. Voisin, and J. M. Gérard, Phys. Rev. B 63, 233306 (2001).

38 H. Saito, K. Nishi, S. Sugou, and Y. Sugimoto, Applied Physics Letters 71, 590 (1997).

39 L. Balet, M. Francardi, A. Gerardino, N. Chauvin, B. Alloing, C. Zinoni, C. Monat, L. H. Li, N. L. Thomas, R. Houdré, and A. Fiore, Appl. Phys. Lett. 91, 123115 (2007).

40 N. Chauvin, P. Nedel, C. Seassal, B. Ben Bakir, X.
Letartre, M. Gendry, A. Fiore, and P. Viktorovitch, Phys. Rev. B 80, 045315 (2009).

41 J. M. Lamy, C. Paranthoen, C. Levallois, A. Nakkar, H. Folliot, J. P. Gauthier, O. Dehaese, A. L. Corre, and S. Loualiche, Appl. Phys. Lett. 95, 011117 (2009).

42 S. Reitzenstein, S. Münch, P. Franeck, A. Löffler, S. Höfling, L. Worschech, A. Forchel, I. V. Ponomarev, and T. L. Reinecke, Phys. Rev. B 82, 121306 (2010). 